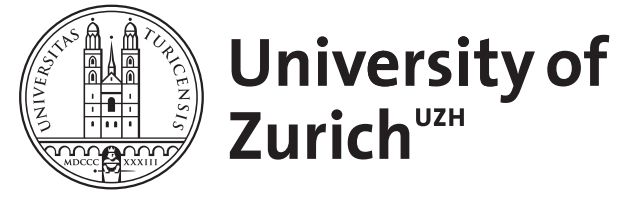
Archive

University of Zurich

University Library

Strickhofstrasse 39

CH-8057 Zurich

www.zora.uzh.ch

Year: 2020

\title{
Poésie et musique chez Baudelaire et Verlaine
}

Klinkert, Thomas

DOI: https://doi.org/10.15203/atem_2020_2.06

Posted at the Zurich Open Repository and Archive, University of Zurich

ZORA URL: https://doi.org/10.5167/uzh-190686

Journal Article

Published Version

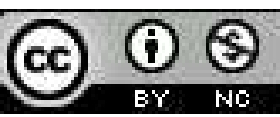

The following work is licensed under a Creative Commons: Attribution-NonCommercial 4.0 International (CC BY-NC 4.0) License.

Originally published at:

Klinkert, Thomas (2020). Poésie et musique chez Baudelaire et Verlaine. ATeM Archives of Text and Music Studies, (5,2):1-16.

DOI: https://doi.org/10.15203/atem_2020_2.06 


\title{
Poésie et musique chez Baudelaire et Verlaine
}

\author{
Thomas KLINKERT (Zurich) ${ }^{1}$
}

\section{Summary}

This article investigates the relationship between poetry and music using Baudelaire's examination of Richard Wagner's music as an example. Starting from the question of the 'translatability' of music into language, which Baudelaire himself poses on the basis of the Lohengrin prelude, the article fundamentally questions the possibility of an association between the two arts, arguing that such an association, if it does not want to remain purely metaphorical, seems possible at best in the area of a 'contact zone', in which the logical-semantic references recede into the background or assume a high degree of generality, while at the same time formal structures can be designed in such a way that the abstract statements are thereby 'represented'. This is illustrated by the example of two poems by Baudelaire ("La musique") and Verlaine (from Romances sans paroles).

\section{Baudelaire et la tentative de ‘ traduire > la musique de Wagner}

L'esthétique de la modernité développée par Baudelaire au milieu du XIX ${ }^{\mathrm{e}}$ siècle a son point de départ dans une perspective résolument intermédiale. Comme on sait, Baudelaire, avant d'être l'auteur des Fleurs du mal (1857), s'est fait connaître comme critique d'art ; son écrit théorique le plus célèbre s'intitule Le peintre de la vie moderne (1863 ; Baudelaire 1976, 683-724). Dans ce texte, il explique sa conception de la modernité à partir des dessins de Constantin Guys, alors que c'est depuis l'année 1846 qu'il emploie l'adjectif « moderne , dans ses textes de critique d'art. ${ }^{2}$ La dimension intermédiale est également en jeu, lorsqu'il est question du rapport entre poésie et musique. Or, comparé au grand nombre d'écrits consacrés à l'art et à la peinture, la réflexion baudelairienne sur la musique semble peu importante. Cependant, le $1^{\mathrm{er}}$ avril 1861, il publiait, dans la Revue européenne, un texte de critique musicale intitulé Richard Wagner et Tannhäuser à Paris (Baudelaire 1976, 779-808). Dans ce texte, " un de ses textes les plus denses et les plus complets quant à la philosophie, la poétique et l'esthétique» (Kopp 1994, XXXVI), on peut lire la phrase suivante : 
[...] si, par le choix de ses sujets et sa méthode dramatique, Wagner se rapproche de l'antiquité, par l'énergie passionnée de son expression il est actuellement le représentant le plus vrai de la nature moderne. (Baudelaire 1976, 806)

En employant le binôme antiquité/modernité pour caractériser l'art musical de Richard Wagner, Baudelaire prête une importance capitale à l'esthétique de ce compositeur, qu'il fait apparaître comme une expression de ses propres principes poétologiques et esthétiques. ${ }^{3}$ Tout en admettant que Baudelaire avait peu de connaissances spécialisées en musique, on doit néanmoins reconnaître la grande sensibilité qui lui permettait de comprendre la musique de Wagner, peu connue en France à cette époque, et qui le portait à faire cet aveu dans une lettre adressée au compositeur le 17 février 1860 : "Avant tout, je veux vous dire que je vous dois la plus grande jouissance musicale que jaie jamais éprouvée. " (Baudelaire 1976, 1452 ; italiques dans le texte)

Dans son article sur Wagner, Baudelaire rend compte de ses impressions d'auditeur et de sa propre expérience, faisant valoir cette subjectivité comme principe d'écriture dès le début du texte, de façon programmatique : « [...] qu'il me soit permis, dans cette appréciation, de parler souvent en mon nom personnel» (Baudelaire 1976, 779).

Un des concepts-clés employés par Baudelaire pour caractériser la musique est celui de la $<$ traduction $>$ :

J'ai souvent entendu dire que la musique ne pouvait pas se vanter de traduire quoi que ce soit avec certitude, comme fait la parole ou la peinture. Cela est vrai dans une certaine proportion, mais n'est pas tout à fait vrai. Elle traduit à sa manière, et par les moyens qui lui sont propres. Dans la musique, comme dans la peinture et même dans la parole écrite, qui est cependant le plus positif des arts, il y a toujours une lacune complétée par l'imagination de l'auditeur. (Baudelaire 1976, 781-782)

Dans ce passage, Baudelaire met sur le même plan la musique, la peinture et la littérature, en avançant que dans chaque réalisation artistique, il reste toujours une part de non-dit, une lacune que doit compléter l'imagination de l'auditeur. Outre que cette remarque fait penser à l'analyse du processus de la lecture proposée par Wolfgang Iser ${ }^{4}$ dans les années 1970, témoignant ainsi d'une indubitable compétence sémiotique, elle présuppose que toute forme d'art peut être déchiffrée jusqu'à un certain degré, c'est-à-dire qu'il y a une signification, autrement dit que la musique - comme les autres arts - peut contenir des idées :

[...] il reste encore incontestable que plus la musique est éloquente, plus la suggestion est rapide et juste, et plus il y a de chances pour que les hommes sensibles conçoivent des idées en rapport avec celles qui inspiraient l'artiste. (Baudelaire 1976, 782)

Pour illustrer cette conception d'un art musical " éloquent ", Baudelaire donne trois ' traductions > verbales de l'ouverture de Lohengrin. ${ }^{5}$ La première mise en parole est em- 
pruntée au programme du Théâtre-Italien où eurent lieu les concerts donnés par Wagner en $1860 .{ }^{6}$ Le texte de ce programme est de la plume de Wagner. La deuxième traduction de ce morceau est de Franz Liszt, célèbre pianiste, compositeur et ami de Wagner qui a essayé d'introduire sa musique en France, et la troisième de Baudelaire lui-même.

Quelles sont les idées que l'audition de Lohengrin a suscitées en Baudelaire ? Voici un extrait de son texte :

Je me souviens que, dès les premières mesures, je subis une de ces impressions heureuses que presque tous les hommes imaginatifs ont connues, par le rêve, dans le sommeil. Je me sentis délivré des liens de la pesanteur, et je retrouvai par le souvenir l'extraordinaire volupté qui circule dans les lieux hauts (notons en passant que je ne connaissais pas le programme cité tout à l'heure). Ensuite je me peignis involontairement l'état délicieux d'un homme en proie à une grande rêverie dans une solitude absolue, mais une solitude avec un immense horizon et une large lumière diffuse ; l'immensité sans autre décor qu'elle-même. Bientôt j'éprouvai la sensation d'une clarté plus vive, d'une intensité de lumière croissant avec une telle rapidité, que les nuances fournies par le dictionnaire ne suffiraient pas à exprimer ce surcroît toujours renaissant d'ardeur et de blancheur. Alors je conçus pleinement l'idée d'une âme se mouvant dans un milieu lumineux, d'une extase faite de volupté et de connaissance, et planant au-dessus et bien loin du monde naturel. (Baudelaire 1976, 784-785 ; italiques Ch.B.)

En étudiant ce passage, on constate que dans chaque phrase l'auteur emploie des expressions qui renvoient à la subjectivité d'un observateur ("je subis une de ces impressions heureuses ", "je me sentis délivré des liens de la pesanteur ", " je me peignis involontairement l'état délicieux d'un homme ", "jéeprouvai la sensation d'une clarté plus vive, d'une intensité de lumière ", "je conçus pleinement l'idée d'une âme se mouvant dans un milieu lumineux "; italiques Th.K.). Par conséquent, il est impossible au lecteur de Baudelaire d'ignorer le fait qu'entre la musique de Wagner et sa traduction verbale il y a un sujet sensible qui reçoit des impressions, qu'il transforme en idées avant de les traduire en un texte verbal. C'est par cet aspect que la < traduction > de Baudelaire se distingue clairement des deux autres qu'il cite dans son texte. Dans le programme du Théâtre-Italien on lit ceci :

Dès les premières mesures, l'âme du pieux solitaire qui attend le vase sacré plonge dans les espaces infinis. Il voit se former peu à peu une apparition étrange qui prend un corps, une figure. Cette apparition se précise davantage, et la troupe miraculeuse des anges, portant au milieu d'eux la coupe sacrée, passe devant lui. Le saint cortège approche ; le cœur de l'élu de Dieu s'exalte peu à peu; il s'élargit, il se dilate; d'ineffables aspirations s'éveillent en lui ; il cède à une béatitude croissante, en se trouvant toujours rapproché de la lumineuse apparition, et quand enfin le Saint-Graal lui-même apparait au milieu du cortège sacré, il śabime dans une adoration extatique, comme si le monde entier eût soudainement disparu. (Baudelaire 1976, 782 ; italiques Ch.B.) 
Ce passage, qui est de Wagner, met l'accent dès le début sur l'action, donc sur le plan diégétique de l'opéra. Il est question du "vase sacré ", c'est-à-dire du Saint-Graal, et donc des circonstances et du milieu d'où est issu le protagoniste de l'opéra, le chevalier Lohengrin. À en croire cette description, la musique de l'ouverture signifie ce que le texte évoque, comme si la musique instrumentale avait un lexique et pouvait renvoyer directement à une réalité extra-musicale de façon mimétique. C'est de façon comparable que procède le deuxième texte cité par Baudelaire, de la plume de Franz Liszt :

Cette introduction renferme et révèle l'élément mystique, toujours présent et toujours caché dans la pièce... Pour nous apprendre l'inénarrable puissance de ce secret, Wagner nous montre d'abord la beauté ineffable du sanctuaire, habité par un Dieu qui venge les opprimés et ne demande qu'amour et foi à ses fidèles. Il nous initie au Saint-Graal [...]. Il ne nous le fait point apercevoir dans son imposante et réelle structure, mais, comme ménageant nos faibles sens, il nous le montre d'abord reflété dans quelque onde azurée ou reproduit par quelque nuage irisé. (Baudelaire 1976, 783 ; italiques Ch.B.)

Tout en nous expliquant, à l'instar de Wagner, la musique selon le schéma ' $\mathrm{X}$ signifie $\mathrm{Y}$, Liszt intègre néanmoins dans sa description le rapport entre Wagner et le public, en disant par exemple : "Wagner nous montre d'abord la beauté ineffable du sanctuaire. "Dans la suite de son texte, Liszt focalise aussi sur le fonctionnement technique de la musique, tout en utilisant un style métaphorique :

C'est au commencement une large nappe dormante de mélodie, un éther vaporeux qui sétend, pour que le tableau sacré s'y dessine à nos yeux profanes ; effet exclusivement confié aux violons, divisés en huit pupitres différents, qui, après plusieurs mesures de sons harmoniques, continuent dans les plus hautes notes de leurs registres. Le motif est ensuite repris par les instruments à vent les plus doux ; les cors et les bassons, en s'y joignant, préparent l'entrée des trompettes et des trombones, qui répètent la mélodie pour la quatrième fois, avec un éclat éblouissant de coloris, comme si dans cet instant unique l'édifice saint avait brillé devant nos regards aveuglés, dans toute sa magnificence lumineuse et radiante. (Baudelaire 1976, 783; italiques Ch.B.)

Dans ce passage, Liszt traduit des aspects de la composition musicale (la mélodie, l'instrumentation) en un équivalent verbal dans le but de justifier la sémantisation qu'il en fait dériver. On remarque l'emploi de métaphores dans cette traduction, par exemple lorsqu'il est question d' " une large nappe dormante de mélodie " ou d' "un éther vaporeux qui s'étend ", ou encore d'un "tableau sacré [qui] s'y dessine à nos yeux profanes". Ainsi le texte de Liszt cité par Baudelaire met à nu les différents processus de transformation sémiotique qui partent de la texture musicale pour lui attribuer des valeurs sémantiques. Dans la dernière phrase citée, il se trouve un élément particulièrement intéressant, à savoir le "comme si " qui rend compte du fait qu'il s'agit d'une interprétation plus ou moins subjective (« comme 
si dans cet instant unique l'édifice saint avait brillé devant nos regards aveuglés, dans toute sa magnificence lumineuse et radiante »). En faisant précéder les signifiés attribués à la texture musicale par la conjonction "comme si ", qui renvoie à quelque chose d'irréel, indiquant par là le côté imaginaire et fictif de ce contenu, le texte de Liszt nous fait comprendre que la sémantisation proposée par l'auteur est hypothétique et donc subjective.

Baudelaire, quant à lui, renforce l'élément subjectif et imaginaire qui s'associe à ce processus d'interprétation et de sémantisation. En comparant les trois traductions qu'il vient de juxtaposer, il croit pouvoir constater qu'il y a entre elles certaines différences, mais aussi de nombreuses ressemblances :

Dans les trois traductions nous trouvons la sensation de la béatitude spirituelle et physique; de l'isolement; de la contemplation de quelque chose infiniment grand et infiniment beau; d'une lumière intense qui réjouit les yeux et l'âme jusqu'à la pâmoison; et enfin la sensation de l'espace étendu jusqu'aux dernières limites concevables. (Baudelaire 1976, 785 ; italiques Ch.B.)

Quant à la nature de ces contenus, on doit constater qu'il s'agit d'impressions relativement abstraites et vagues. Il est question de " béatitude ", $\mathrm{d}^{\prime}$ " isolement ", de " lumière intense " et de "l'étendue de l'espace ». On peut en conclure qu'apparemment la sémantisation de la musique ne peut se situer qu'à un niveau élevé d'abstraction. Malgré les affirmations réitérées selon lesquelles la musique peut peindre quelque chose, elle est plutôt apte à provoquer des impressions et des sensations générales comme celles qui viennent d'être évoquées et qui par la suite peuvent donner lieu à une traduction plus spécifique opérée par l'auditeur. D'ailleurs, il y a chez Baudelaire la conscience que cette traduction est par moments guidée par la subjectivité radicale d'un observateur, qui serait sous l'influence de narcotiques susceptibles d'augmenter sa capacité d'imagination, voire d'hallucination. Ainsi il dit : « Il semble parfois, en écoutant cette musique ardente et despotique, qu'on retrouve peintes sur le fond des ténèbres, déchiré par la rêverie, les vertigineuses conceptions de l'opium. " (Baudelaire 1976, 785) La musique est donc identifiée à l'opium, dont Baudelaire parle dans les Paradis artificiels (cf. à ce sujet Klinkert 2018).

On voit clairement que l'auteur des Fleurs $d u$ mal cherche à trouver dans la musique une confirmation de ses convictions esthétiques et poétologiques. ${ }^{7}$ Dans les réflexions précédant la < traduction > que Baudelaire donne des impressions qu'il avait reçues en écoutant l'ouverture de Lohengrin, il se sert d'un raisonnement a priori disant que " ce qui serait vraiment surprenant, c'est que le son ne pût pas suggérer la couleur, que les couleurs ne pussent pas donner l'idée d'une mélodie, et que le son et la couleur fussent impropres à traduire des idées " (Baudelaire 1976, 784 ; italiques Ch.B.). Pour illustrer cette conception d'harmonie universelle, il cite deux strophes de son poème "Correspondances " (Baudelaire 1975, IV, 11). Selon cette conception, "Les parfums, les couleurs et les sons se répondent ", c'est-àdire qu'ils s'évoquent mutuellement, que les sons, les couleurs, les parfums sont des signes non arbitraires, quasi naturels, qui se font écho. Il s'y exprime une conception cratyliste (cf. 
Genette 1976), selon laquelle les signes du langage, les signes de l'art et les signes de la nature font partie d'un système universel, naturel et harmonieux, non arbitraire, d'un monde sensible et compréhensible. Sur ce fond, il n'est pas étonnant que Baudelaire croie percevoir une analogie profonde entre la musique et le langage, analogie qu'il vient d'exprimer dans ses réflexions sur Wagner en traduisant les impressions causées par la musique et en harmonisant les trois traductions, pourtant assez différentes, qu'il juxtapose.

\section{La rivalité entre musique et poésie}

Que cela nous apprend-il en vue du rapport entre musique et poésie ? ${ }^{8}$ Comme on sait, Wagner combinait l'art littéraire et l'art musical pour en faire ce qu'il appelle ‘ l'œuvre d'art totale , Gesamtkunstwerk). Par conséquent, Baudelaire parle de la complémentarité de l'art musical et de l'art poétique chez Wagner en disant qu' « il lui fut impossible de ne pas penser d'une manière double, poétiquement et musicalement, de ne pas entrevoir toute idée sous deux formes simultanées, l'un des deux arts commençant sa fonction là où s'arrêtent les limites de l'autre " (Baudelaire 1976, 787-788). Dans ses écrits, Wagner parle lui-même de l'alliance entre la poésie et la musique, alliance qui, cependant, s'avère aussi être une rivalité. Ainsi, Baudelaire cite une réflexion du compositeur dans laquelle il définit le rapport entre la poésie et la musique de la façon suivante :

Larrangement rythmique et l'ornement (presque musical) de la rime sont pour le poète des moyens d'assurer au vers, à la phrase, une puissance qui captive comme par un charme et gouverne à son gré le sentiment. Essentielle au poète, cette tendance le conduit jusqu’à la limite de son art, limite que touche immédiatement la musique, et, par conséquent, l'œuvre la plus complète du poète devrait être celle qui, dans son dernier achèvement, serait une parfaite musique. (Wagner, cité d'après Baudelaire 1976, 791)

Pour avoir une pleine intelligence de ce que Wagner veut dire, il faut prendre en considération ce qu'il entend par ‘ poétique `. C'est dans le même passage qu'il en donne une définition en disant que ce qui est poétique se définit par la perception des « mobiles purement humains qui gouvernent le cœur " (Wagner, cité d'après Baudelaire 1976, 791). Clairement, cela n'est pas une définition technique du mot ‘ poétique '. Au contraire, Wagner définit le poétique par une situation ayant trait à la vie humaine en général. C'est ce qu'en allemand on appelle "das allgemein Menschliche ", donc le côté anthropologique de la vie humaine. Tous les procédés techniques que peut employer le poète, à savoir l'arrangement rythmique, l'ornement de la rime, etc., doivent, en dernière analyse, viser à captiver le sentiment. Si Wagner parle de la limite de l'art, il veut dire que le poète doit dépasser la dimension sémantique du langage pour atteindre directement les sentiments, chose que peut faire la musique, si bien que dans son dernier achèvement la poésie se confondrait avec la musique. 
Ces réflexions de Wagner se situent sur le fond d'une rivalité entre la poésie et la musique. Lacoue-Labarthe montre que Wagner s'interroge sur la possibilité qu'a l'art « de promouvoir une forme idéale " (Lacoue-Labarthe 1981, 25 ; italiques dans le texte). Cette forme idéale qui serait une forme "universelle ", "supranationale ", "purement humaine " (LacoueLabarthe 1981, 25), c'est la musique qui est seule capable de la produire, car, dit Wagner, " la musique est une langue également intelligible à tous les hommes, et elle devait être la puissance conciliatrice, la langue souveraine, qui, résolvant les idées en sentiments, offrait un organe universel de ce que l'intuition de l'artiste a de plus intime " (Wagner, Quatre poèmes d'opéras, cité d'après Lacoue-Labarthe 1981, 25). Il en résulte que la musique est mise en concurrence avec la littérature qui, elle, « est liée et subordonnée à la diversité des langues naturelles " (Wagner, cité d'après Lacoue-Labarthe 1981, 26). Par un renversement dialectique, la musique, qui peut exprimer la subjectivité, « est à même de dire l'universel » (Wagner, cité d'après Lacoue-Labarthe 1981, 26), ce qui lui donne un avantage considérable par rapport à la poésie qui, elle, doit se servir d'une langue nationale, ce qui lui interdit de devenir universelle, alors qu'en même temps, elle est contrainte de passer par l'abstraction du langage, ce qui l'empêche aussi d'exprimer l'intimité d'un sujet. En outre, Wagner constate que « chaque art tend à une extension indéfinie de sa puissance, que cette tendance le conduit finalement à sa limite, et que, cette limite, il ne saurait la franchir sans courir le risque de se perdre dans l'incompréhensible, le bizarre et l'absurde " (Wagner, cité d'après Lacoue-Labarthe 1981, 27). De là découle la nécessité, selon Wagner, de combiner la musique et la poésie sous forme d'œuvre d'art totale qui est " un moyen de contenir l'excès " (Wagner, cité d'après LacoueLabarthe 1981, 28). Il s'agit notamment de contenir l'excès de la poésie : « La poésie, dans sa tendance même à l'excès, dans le pouvoir transgressif qui est le sien, représente au fond la plus grande menace. » (Wagner, cité d'après Lacoue-Labarthe 1981, 28) Selon Wagner, il y a dans le poète un "penchant naturel " qui consiste à "employer l'instrument des idées abstraites, la langue, de telle sorte qu'elle agisse sur la sensibilité elle-même " (Wagner, cité d'après Lacoue-Labarthe 1981, 28). Il s'agit donc pour le poète de dépasser l'abstraction inscrite dans le langage en inventant un sujet poétique permettant la manifestation des « mobiles purement humains qui gouvernent le cœur ». De surcroît, le poète cherchera "à substituer à la valeur abstraite et conventionnelle des mots leur signification sensible et originelle ", ce qui passe par "l'arrangement rythmique et l'ornement (déjà presque musical) de la rime " (Wagner, cité d'après Lacoue-Labarthe 1981, 28). Autrement dit, le poète qui veut saisir et exprimer la subjectivité a tendance à faire dériver la poésie vers la musique : « l'œuvre la plus complète du poète devrait être celle qui, dans son dernier achèvement, serait une parfaite musique. " (Wagner, cité d'après Lacoue-Labarthe 1981, 28 ; Baudelaire, ainsi que nous l'avons signalé ci-dessus, cite le même passage : 1976, 791) Si la poésie, selon Wagner, tend vers la musique, celle-ci, en revanche, à travers son évolution se transforme en langage.

Face à cette situation, la poésie a en principe deux possibilités. Elle peut s'assimiler à la philosophie en passant dans « le champ de l'abstraction, de la pure combinaison des idées, de la représentation du monde au moyen des lois logiques de la pensée ", ou bien la poésie peut "se fondre intimement avec la musique " pour "se résoudre finalement dans la musique " 
(Wagner, cité par Lacoue-Labarthe 1981, 31). C'est cette deuxième solution qu'adopte Wagner lui-même en concevant la poésie comme élément constitutif de l'opéra de l'avenir, tel qu'il s'apprête à le créer et dans lequel « elle aura pour fonction de présenter l'idée de manière sensible, c'est-à-dire l'idée comme mythe " (Lacoue-Labarthe 1981, 31).

En d'autres mots : les idées de Wagner, qui postulent la supériorité de la musique, constituent un énorme défi pour les poètes, défi qui ne peut manquer d'inquiéter ces derniers. Or, ainsi que le montre Lacoue-Labarthe dans son analyse de la réaction de Baudelaire, celui-ci acquiesce et se soumet au postulat wagnérien de la supériorité de la musique, ce qui s'exprime entre autres par le fait que dans son essai sur Wagner, il revendique la subjectivité de sa propre expérience musicale en établissant un rapport communicatif avec Wagner, rapport qui met Baudelaire dans la position de quelqu'un qui demanderait à la musique du compositeur allemand de le constituer comme sujet : "c'est un sujet qui attend de la musique de Wagner son «moyen d'identification ». " (Lacoue-Labarthe 1981, 34) On peut notamment constater, et Lacoue-Labarthe n'est pas le seul à l'avoir fait (cf. la note 2 du présent article), que Baudelaire retrouve chez Wagner les éléments saillants de sa propre poétique, à savoir l'anamnèse, le sublime, l'élévation, l'extase, le culte de la sensation, l'imagination, les correspondances, le bizarre et les excitants (Lacoue-Labarthe 1981, 36). En se soumettant de cette manière à Wagner, Baudelaire s'approprie l'idée wagnérienne selon laquelle « la musique a désormais pris, définitivement, le relais de la poésie" (Lacoue-Labarthe 1981, 43). Néanmoins, tout en paraissant admettre la supériorité de la musique, Baudelaire détourne cette esthétique musicale en la coulant dans le moule de sa propre esthétique, qui est une esthétique poétique et littéraire. L'un des éléments fondateurs de cette poétique est la dualité de l'homme, qui en fait un être foncièrement déchiré : "Il n'y a pas d'unification possible du sujet : c'est l'enfer ou le ciel, mais jamais l'un comme l'autre. " (Lacoue-Labarthe 1981, 50 ; italiques dans le texte) En interprétant Tannhäuser, Baudelaire insiste sur l'élément satanique, créditant ainsi Wagner "d'une sorte de mysticisme ( négatif > qui est celui des Fleurs du mal ou des ‘ Journaux intimes `. Manifestement, ce que reconnaît Baudelaire dans Wagner, c'est ce qui n'y est pas : non pas la rédemption, mais un absolu atteint, touché dans le mal - c'est-à-dire aussi bien dans la plus grande perte de soi » (Lacoue-Labarthe 1981, 50 ; italiques dans le texte). Par conséquent, on peut dire avec Lacoue-Labarthe que, tout en semblant scrupuleusement se plier à la logique wagnérienne, Baudelaire soumet celle-ci à sa propre logique, amenant ainsi une "perversion de Wagner " (Lacoue-Labarthe 1981, 50 ; italiques dans le texte). Cette " perversion " repose sur une conception selon laquelle la musique de Wagner possède un caractère systématique ressemblant par là même à la poésie, étant donné qu'elle est explicative " par elle-même » du fait de la concaténation des éléments qui la composent (cf. Baudelaire 1976, 803). Selon Baudelaire la musique de Wagner est poésie et donc écriture, voire livre. (Lacoue-Labarthe 1981, 52)

Si Wagner veut créer une poésie qui dépasse la dimension du sens, donc le côté sémantique du langage, pour aller directement au sentiment, Baudelaire, quant à lui, conçoit la musique comme un moyen d'expression qui est une sorte de langage, une poésie. Il veut démontrer " que la véritable musique suggère des idées analogues dans des cerveaux diffé- 
rents " (Baudelaire 1976, 784). L'endroit où les deux conceptions se rencontrent, c'est le niveau de l'abstraction, celui des sentiments généraux, de ce que tout homme peut sentir et comprendre. Je propose d'appeler cette sphère une ‘ zone de contact > entre musique et poésie. C'est dans cette zone de contact que les deux arts peuvent se rencontrer (cf. infra).

\section{Une poésie ‘ musicale ` ? (Baudelaire et Verlaine)}

Cependant, on peut se demander comment un texte poétique doit être structuré afin de se rapprocher de la musique. Si l'on compare systématiquement la musique et la poésie, on constate qu'il s'agit de deux systèmes sémiotiques foncièrement différents (cf. l'Introduction du présent volume), si bien que toute identification se fondant sur la perception de la musique comme un langage ne peut être que métaphorique. La musique n'est pas un langage au sens strict, car elle n’a pas de dimension sémantique. En revanche, la poésie peut se rapprocher de la musique dans la mesure où le langage peut être chanté. Entre le langage et la musique il y a donc une ( zone de contact ) que certains poètes mettent à profit pour créer des formes poétiques originales. Celles-ci se caractérisent par une corrélation, voire une adéquation entre la structuration formelle et le niveau sémantique.

Dans les ouvres poétiques de Baudelaire, on trouve quelques références à la musique, notamment dans le sonnet " La musique " contenu dans Les fleurs du mal, mais aussi dans la dédicace du Spleen de Paris. Considérons brièvement "La musique " (Baudelaire 1975, LXIX, 68) :

La musique souvent me prend comme une mer!

Vers ma pâle étoile,

Sous un plafond de brume ou dans un vaste éther,

Je mets à la voile ;

La poitrine en avant et les poumons gonflés

Comme de la toile,

J'escalade le dos des flots amoncelés

Que la nuit me voile;

Je sens vibrer en moi toutes les passions

D'un vaisseau qui souffre ;

Le bon vent, la tempête et ses convulsions

Sur l'immense gouffre

Me bercent. D'autres fois, calme plat, grand miroir

De mon désespoir! 
Comme dans la (traduction , de l'ouverture de Lohengrin, dans ce poème l'accent est mis sur les effets que produit la musique sur le sujet. La puissance de ces effets est rendue moyennant la métaphore de la mer qui est ici employée en tant que métaphore filée. Le sujet de l'énonciation se trouve exposé sans aucune protection à cette puissance de la mer, "[l]a poitrine en avant et les poumons gonflés ". Saisi par la mer, le sujet, transformé métaphoriquement en un bateau, subit passivement les conséquences de cette force. Il sent « vibrer » en lui « toutes les passions / D'un vaisseau qui souffre ». Face à la force de la musique, le sujet peut ressentir des "passions ", mais aussi du " désespoir ». La mer est pour lui comme un "miroir ». Si l'on admet que le voyage en mer, en accord avec une tradition ancienne (cf. Curtius ${ }^{11} 1993,138-140$ ), est une métaphore poétologique, on peut assumer que les effets de la musique sur le bateau ont également une valeur poétologique. La musique "berce " le sujet devenu bateau " [s] ur l'immense gouffre ». Qui plus est, les " convulsions " de la " tempête " se réfléchissent dans la structure même de ce sonnet hétérométrique, qui est composé d'alexandrins et de pentasyllabes; à l'irrégularité de la structure syllabique s'ajoute une autre anomalie, à savoir la répétition de la deuxième rime dans les deux quatrains qui suivent le schéma : $\mathrm{A}-\mathrm{b}-\mathrm{A}-\mathrm{b} / \mathrm{C}-\mathrm{b}-\mathrm{C}-\mathrm{b}$. Ce sonnet est donc marqué par des « convulsions " formelles. C'est ainsi que le poème semble vouloir intégrer dans sa structure même la violence subie par la musique, identifiée à la mer. Nous avons donc affaire à un phénomène d'analogie entre des éléments de sens (dimension sémantique) et des éléments formels (dimension métrique du poème). Une telle analogie est un trait typique des textes poétiques; il s'agit d'une tentative de faire apparaittre les signes linguistiques, qui sont en principe arbitraires, comme motivés, ce que l'on peut appeler, avec Gérard Genette, un cratylisme secondaire (cf. Genette 1976). Et ce n'est peut-être pas un hasard si Baudelaire expose ce principe de façon particulièrement transparente dans un poème consacré à la musique.

Un autre exemple de poésie (musicale ) est à considérer ici, à savoir les Romances sans paroles de Paul Verlaine. ${ }^{9}$ Le titre de ce recueil renvoie explicitement au domaine de la musique, dans la mesure où il reprend le titre d'une ouvre musicale de Mendelssohn. ${ }^{10} \mathrm{C}$ 'est un recueil de pièces pour piano qui en allemand s'intitule Lieder ohne Worte. Une chanson sans paroles est littéralement un paradoxe, car c'est par la parole même que se définit le chant. Si le compositeur renonce à un élément constitutif du chant, à savoir la dimension verbale, il renforce la dimension musicale produisant en quelque sorte une version ‘ plus pure ', ' plus absolue > de cette forme musicale. Il s'agit de Lieder qui ont une mélodie et que l'on pourrait chanter si on disposait de paroles. Ce qui a lieu, c'est donc un processus de défamiliarisation. C'est également un processus de défamiliarisation que Verlaine fait subir à la poésie en empruntant le titre musical à Mendelssohn. Le paradoxe est ainsi renforcé car si on peut à la rigueur imaginer une chanson sans paroles, une poésie n'est certes pas concevable sans paroles. Le titre est donc tout à fait métaphorique, mais il indique clairement que la poésie de Verlaine se propose de mener un dialogue avec la musique.

Il est sans doute un peu exagéré d'affirmer avec Jacques Borel que «c'est une langue poétique essentiellement musicale que l'art verlainien allait à créer. Cette traduction immédiate 
du senti, c'est la musique qui, avant toute image, la communique. [...] Partout ici les mots sont notes. La trame du poème est purement mélodique, et, à un moindre degré, harmonique ; la logique même du poème, sa logique intime, profonde, n'est jamais conceptuelle, mais musicale. " (Verlaine 1962,181) Les poèmes du recueil ne sont pas des morceaux de musique. Ils emploient le langage et reposent par conséquent sur une logique conceptuelle. Il est donc impossible que la trame d'un poème soit purement mélodique ou harmonique. Néanmoins, on constate que dans ces poèmes, le sujet de l'énonciation, qui mène un dialogue avec un ‘ tu > qu'il aime mais qui semble l'avoir abandonné, emploie le langage d'une façon radicalement subjective, dans la mesure où toute perception d'un monde extérieur est immédiatement traduite en états d'âme. Considérons à titre d'exemple la deuxième "Romance sans paroles":

Je devine, à travers un murmure, Le contour subtil des voix anciennes Et dans les lueurs musiciennes, Amour pâle, une aurore future!

Et mon âme et mon cour en délires

Ne sont plus qu'une espèce d'œil double

Où tremblote à travers un jour trouble

Lariette, hélas ! de toutes lyres !

Ô mourir de cette mort seulette

Que s'en vont, - cher amour qui t'épeures, -

Balançant jeunes et vieilles heures!

Ô mourir de cette escarpolette !

(Verlaine 1962, 191-192)

Le poème commence par une perception du sujet qui n'est pas rattachée à des phénomènes clairement définis. Le sujet entend un "murmure " dont la nature n'est pas précisée et ce murmure semble évoquer des souvenirs puisqu'il est question de "voix anciennes "; ces souvenirs semblent à leur tour être en relation avec une impression musicale qui, elle, se manifeste à travers une perception synesthésique : les "lueurs ", perception visuelle, sont associées à une perception acoustique, elles sont " musiciennes ». De même, le passé et le futur sont intimement liés car c'est dans ce souvenir "deviné " par le sujet que celui-ci croit apercevoir une " aurore future ». Or, si le passé et l'avenir peuvent être considérés comme des éléments équivalents, cela correspond à un bouleversement des rapports sémantiques usuels. Au début du vers 4, il y a un élément dont la fonction syntaxique et sémantique est difficile à déterminer : "Amour pâle ». S'agit-il d'une spécification anaphorique ou cataphorique, autrement dit, l'" Amour pâle » se rapporte-t-il aux « lueurs musiciennes " ou à " l'aurore future " ? Ou bien s'agit-il de la même entité qui, au v. 10, fait l'objet d'une apostrophe ("cher amour qui t'épeures») ? 
Dans la deuxième strophe, le sujet parle de son "âme " et de son " cœur " qui sont " en délires ", donc dans un état exceptionnel. Il est hors de lui, ce qui explique sa perception idiosyncrasique dont il a déjà été question dans la première strophe. Cette perception idiosyncrasique s'exprime dans la deuxième strophe à travers l'identification de l'âme et du cœur avec un " œil double». Or, la perception de cet œeil n'est pas visuelle mais acoustique. Il s'agit donc à nouveau d'une perception synesthésique - l'" œil double " aperçoit une " ariette ", donc un phénomène acoustique -, mais il faut ajouter qu'elle n'est pas claire et distincte, car cette perception passe «à travers un jour trouble ». Qui plus est, ce que cet œil aperçoit n'est pas simplement une ariette, mais c'est une ariette au superlatif, l'ariette "de toutes lyres". Il s'agit donc d'un phénomène d'intensification qui semble provoquer une douleur, car le sujet exprime un regret en disant " hélas! ». Dans la troisième strophe, le sujet manifeste le désir de mourir. Ce désir n’est cependant pas énoncé par un sujet qui dirait clairement « je veux mourir ", il s'agit d'un désir impersonnel, un désir sans sujet. Cette troisième strophe se caractérise par un flou sémantique et syntaxique, puisqu'il n'y a pas de sujet grammatical et que les rapports sémantiques sont loin d'être clairs : que signifie l'idée que les « jeunes et vieilles heures » « s'en vont » « [b]alançant » une « mort seulette » ?

Ainsi, ce poème nous présente un sujet dont les perceptions sont extrêmement incertaines. En fait, on ne sait aucunement dans quel monde est situé ce sujet et quels éléments de ce monde il aperçoit. Nous savons que ce < je > est dans un état de délire, que ses perceptions sont synesthésiques et confuses, qu'il est apparemment au désespoir à cause d'un amour perdu, qu'il a un rapport intime avec la musique, puisqu'il parle de " lueurs musiciennes " et de "l'ariette [...] de toutes lyres ", et qu'il a le désir de mourir. À la désagrégation du sujet en délire correspond un certain flou syntaxique et sémantique du poème, qui résulte, entre autres, de la position syntaxique ambiguë de l'expression "Amour pâle " (v. 4) et du flou sémantique de la troisième strophe. La forme et le contenu sont ainsi mis en homologie, comme dans le poème "La musique " de Baudelaire. Il est question, dans la troisième strophe, d'un mouvement de balançoire (" escarpolette ») qui peut être rattaché au délire dont il est question dans la strophe précédente, et ce mouvement de balançoire s'associe au désir, exprimé de façon impersonnelle, de mourir. Il y a donc dans ce poème, comme dans les autres poèmes du recueil de Verlaine, un relâchement des rapports logiques, syntaxiques et sémantiques, ce qui donne lieu à un certain flou, à une réduction de la dimension mimétique, réduction qui favorise la perception du matériau verbal et sonore de ce texte, rendant perceptible l'homologie entre la forme et le contenu. C'est ainsi que le texte poétique se situe dans la zone de contact séparant la poésie de la musique, faisant passer la dimension sémantique à l'arrière-plan et créant une forme d'expression qui possède une certaine équivalence avec la musique. 


\section{Conclusion}

Si la musique et la poésie sont deux systèmes sémiotiques différents, elles peuvent se rencontrer dans une zone de contact qui existe depuis bien longtemps, dans la mesure où la musique et le chant ont toujours coexisté, c'est-à-dire que la musique possède la faculté de traduire le langage en un équivalent sonore qui en exprime certaines valeurs. L'idée d'une traduction de pensées en musique qui s'exprime dans l'essai de Baudelaire consacré à Wagner se situe dans un contexte historique bien spécifique, qui est caractérisé par la rivalité entre la poésie et la musique. Mis à part le phénomène spécifique et rare de l'imitation onomatopoétique (que l'on pense, par exemple, à la Symphonie pastorale de Beethoven où la musique imite le chant des oiseaux ou bien un orage), les structures musicales qui cherchent à traduire des valeurs sémantiques se situent à un niveau relativement abstrait et général. Par exemple, dans le célèbre lied de Schubert intitulé «Der Tod und das Mädchen » "La jeune fille et la mort »), le chant de la jeune fille qui a peur de mourir se caractérise par un rythme rapide et haletant, exprimant l'angoisse, alors que le thème de la mort avec lequel commence le lied et qui est repris dans sa partie finale est un thème lent et tranquille, une espèce de choral, qui souligne le message consolateur chanté par la mort qui affirme : "Bin Freund, und komme nicht zu strafen. / Sei gutes Muts ! Ich bin nicht wild, / Sollst sanft in meinen Armen schlafen! » La mélodie de la jeune fille se distingue de celle chantée par la mort par son rythme et la ligne mélodique. La différence entre les deux mélodies peut être saisie par des termes comme ( stabilité >, ‘ continuité >, ‘ monotonie > versus ( mouvement >, ‘ changement , ' variation `. Ces caractéristiques de la musique, d'abord abstraites, reçoivent leurs valeurs sémantiques définitives par le texte. Si, inversement, la poésie veut se rapprocher de la musique, comme c'est le cas dans les Romances sans paroles de Verlaine, le poète doit, lui aussi, employer des procédés qui ont une portée générale.

Un poème, à moins d'être chanté, n'est jamais de la musique mais il peut faire semblant d'imiter la musique en créant des structures qui peuvent être considérées comme des équivalents de structures musicales. Dans les exemples que nous avons analysés, cette équivalence se situe au niveau des " convulsions ", qui touchent à la fois le sujet confronté à la musique et la forme du poème chez Baudelaire, et, chez Verlaine, au niveau de la désintégration du sujet, qui est un sujet en délire à la perception incertaine, ce qui donne lieu à un flou sémantique. Cette impression de désagrégation est renforcée par des ambiguïtés syntaxiques, si bien que nous y retrouvons un signe motivé, c'est-à-dire un signe dont la structure formelle fait écho à la signification. Bien entendu, cette structure reste foncièrement verbale et linguistique. Paradoxalement, c'est en évoquant le modèle musical que le texte poétique fait valoir les propriétés les plus caractéristiques du langage. Le rapprochement de la poésie à la musique ne peut être qu'une suggestion ou une évocation; il n'est jamais réel au sens technique. Néanmoins, les exemples que nous avons étudiés dans cette contribution montrent que c'est en se confrontant à la musique que les poètes novateurs du XIX ${ }^{\mathrm{e}}$ siècle se rendent compte de ce que la poésie doit avoir de spécifique. 


\section{Notes}

1 Thomas Klinkert est professeur de littérature française moderne à l'Université de Zurich. Spécialiste d'épistémocritique et de théorie de la littérature, il a notamment travaillé sur Dante, Rousseau, Diderot, Baudelaire, Pirandello, Proust et Claude Simon. Il est l'auteur de plusieurs livres, parmi lesquels Epistemologische Fiktionen. Zur Interferenz von Literatur und Wissenschaft seit der Aufklärung (2010), Muße und Erzählen - ein poetologischer Zusammenhang. Vom "Roman de la Rose " bis zu Jorge Semprún (2016) et Fiktion, Wissen, Gedächtnis. Literaturtheoretische Studien (à paraître en 2020).

2 Le dernier chapitre du Salon de 1846 (Baudelaire 1976, 415-496) est intitulé " De l'héroïsme de la vie moderne » (Baudelaire 1976, 493-496) et comporte une réflexion sur la dualité de la beauté, qui contient "quelque chose d'éternel et quelque chose de transitoire » (493), ce qui anticipe la célèbre " théorie rationnelle et historique du beau » du Peintre de la vie moderne (Baudelaire 1976, 685), selon laquelle « le beau est toujours, inévitablement, d'une composition double ", comprenant " un élément éternel, invariable » et " un élément relatif, circonstanciel » (Baudelaire 1976, 685).

3 Le rapport entre Baudelaire et Wagner eu égard à l'esthétique moderne a souvent été analysé par la critique. Ainsi, pour citer quelques exemples, selon Kopp, Baudelaire « a reconnu en Wagner comme dans Delacroix ou Poe - son propre modernisme et son propre romantisme, un romantisme fait d'une spiritualité qui confine au mysticisme " (Kopp 1994, XLV). Ainsi que l'affirme Jackson, "le poète français avait reconnu en Wagner l'image de son propre idéal de créateur " (Jackson 2001, 209). « Pour Baudelaire, Richard Wagner offre la conception du nouvel opéra, ou plutôt de ce qui sera bientôt le drame musical, où à la conception ancienne d'une ‘ déclamation notée et soulignée par la musique > succède celle, véritablement moderne, d'une ‘ totalité d'effet `. " (Brunel 2015, 83)

4 Selon Iser (1979), les textes littéraires se caractérisent par un certain degré d'indétermination ("Unbestimmtheit») et par des lacunes ("Leerstellen») que le lecteur est appelé à remplir en accomplissant l'acte de lecture.

5 Pour une analyse du rapport entre Baudelaire et Wagner, cf., entre autres, Böschenstein (1999), Breatnach (2002), Dayan (2000), Touya de Marenne (2001), Landi (2019).

6 Kopp (1994, XXVII-XXVIII) indique le programme de ces concerts, qui eurent lieu le 25 janvier, ainsi que les $1^{\text {er }}$ et 8 février 1860 et qui, dans la première partie, donnèrent à écouter l'Ouverture du Vaisseau fantôme, Marche et chœur, l'Introduction du III ${ }^{e}$ acte, le Chant des pèlerins et l'Ouverture de Tannhäuser, et, dans la deuxième partie, le Prélude de Tristan et Iseult, et, enfin, des extraits de Lohengrin, à savoir l'Introduction, la Marche des fiançailles et la Fête nuptiale (Introduction du III ${ }^{\mathrm{e}}$ acte) et Épithalame.

7 Pour l'analyse du côté circulaire de la ‘ traduction > effectuée par Baudelaire, dont le but ultime est de trouver une confirmation de sa propre esthétique chez Wagner, cf. Dayan $(2000,153)$ : «En un premier temps, il affirme que ce que la musique traduit relève de l'indéfini, n'est pas du ressort de la parole. En un second temps - mais ces temps se confondent - il analyse la traduction en musique en travaillant sur des originaux écrits, qui lui fournissent du < positif > : les trois < tra- 
ductions > sur l'Ouverture, les essais de Wagner, les paroles des opéras ; et dans ce travail, il fait ressortir ce qui, dans ces écrits, tend à nier le positif, à s'envoler vers l'indéfini, le vaste, le tout. Et - troisième temps, par lequel on revient au premier - il reverse ces qualités d'indéfini sur la musique ; comme s'il était arrivé à les en faire sortir par traduction directe. Ce qui n'est tout simplement pas le cas. » (Italiques dans le texte) Par conséquent, Dayan parle d'un «brillant tour de passe-passe » (Dayan 2000, 153), qui sert à " maintenir et à démasquer en même temps la fiction dont la poétique de Baudelaire dépend tout entière : qu'il puisse y avoir un lien garanti entre le défini et l'indéfini, entre le positif et le poétique, entre le fini et l'infini, entre le phénomène et l'idée, et que l'art puisse se définir par son ambition de faire sentir l'existence de ce lien » (Dayan 2000, 153).

8 Pour une première approche des enjeux que comporte ce rapport, cf. les études réunies dans le volume collectif de Scher (1984), ainsi que l'ouvrage de Backès (1994).

9 Pour Verlaine et la musique, cf., entre autres, Gür (1999), Kurakata (2004 et 2007), Lichtenthal (2015).

10 On ne sait pas exactement si Verlaine connaissait les pièces de Mendelssohn. L'éditeur de l'édition de la Pléiade, Jacques Borel, écrit que le titre du recueil a été " [e]mprunté à Mendelssohn sans doute " (Verlaine 1962, 181). Kensaku Kurakata, quant à lui, fait remarquer que "Verlaine ne mentionne nulle part dans son œuvre le nom de Mendelssohn » (Kurakata 2004, 103), tout en admettant qu'il est probable que "le poète au même titre que bon nombre de ses contemporains, connaissait le nom du compositeur ainsi que ses œuvres" (Kurakata 2004, 103). En tout cas, il paraît tout à fait légitime d'interpréter l'identité de titre entre le recueil de poèmes de Verlaine et la partition de Mendelssohn comme un indice permettant, voire suscitant une lecture < intermédiale , et poétologique de ce recueil.

\section{Bibliographie}

Backès, Jean-Louis : Musique et littérature. Essai de poétique comparée. Paris : PUF, 1994.

Baudelaire, Charles : Euvres complètes. Éd. Claude Pichois. Vol. 1. Paris : Gallimard, 1975.

Baudelaire, Charles : Euvres complètes. Éd. Claude Pichois. Vol. 2. Paris : Gallimard, 1976.

Böschenstein, Bernhard : "Wie verwandelt sich Musik in Literatur? Jean Paul, Hoffmann,

Mörike, Baudelaire, Thomas Mann ». In : Daigger, Annette / Schröder-Werle, Renate / Thöming, Jürgen (éds) : West-östlicher Divan zum utopischen Kakanien. Hommage à Marie-Louise Roth. Berne : Peter Lang, 1999, 41-59.

Breatnach, Mary : "Baudelaire, Wagner, Mallarmé. Romantic Aesthetics and the Word-Tone Dichotomy ». In : Lodato, Suzanne M. / Aspden, Suzanne / Bernhart, Walter (éds) : Word and Music Studies. Essays in Honor of Steven Paul Scher and on Cultural Identity and the Musical Stage. Amsterdam/New York : Rodopi, 2002, 69-83.

Brunel, Pierre : "Baudelaire et la musique : moderne ou antimoderne ". In : L'Année Baudelaire 18/19 (2015), 69-88. 
Curtius, Ernst Robert : Europäische Literatur und lateinisches Mittelalter. Tübingen/Basel : Francke, ${ }^{11} 1993$.

Dayan, Peter : "De la traduction en musique chez Baudelaire ». In : Romance Studies 18,2 (2000), 145-155, https://doi.org/10.1179/ros.2000.18.2.145 (consultation 30.03.2020).

Genette, Gérard : Mimologiques. Voyage en Cratylie. Paris : Seuil, 1976.

Gür, Adrien : "Verlaine entre voix anciennes et voix musiciennes ». In : Petris, Loris / Bornand, Marie (éds) : Sources et intertexte : résurgences littéraires du Moyen Âge au XXe siècle. Lausanne : Études de Lettres, 1999, 147-156.

Iser, Wolfgang : «Die Appellstruktur der Texte. Unbestimmtheit als Wirkungsbedingung literarischer Prosa ». In : Warning, Rainer (éd.) : Rezeptionsästhetik. Theorie und Praxis. München : Fink, ${ }^{2} 1979,228-252$.

Jackson, John E. : « Baudelaire et Wagner ou la rencontre de deux modernités ». In : Jackson, John E. : Souvent dans l'être obscur. Rêves, capacité négative et romantisme européen. Paris : Corti, 2001, 209-241.

Klinkert, Thomas : "Intoxication and the Aesthetics of Modern Poetry (Baudelaire, Rimbaud, Mallarmé) ». In : Herlinghaus, Hermann (éd.) : The Pharmakon. Concept Figure, Image of Transgression, Poetic Practice. Heidelberg: Winter, 2018, 223-250.

Kopp, Robert : «Baudelaire et Wagner ». In : Baudelaire, Charles : Richard Wagner et Tannhäuser à Paris. Suivi de textes sur Richard Wagner par Nerval, Gautier et Champlleury. Introd. Robert Kopp. Paris : Les Belles Lettres, 1994, IX-XLVI.

Kurakata, Kensaku : "Vers les « romances sans paroles `. Verlaine et la ( musique > ". In : Etudes de langue et littérature françaises 84 (2004), 103-116, https://doi.org/10.20634/ ellf.84.0_103 (consultation 30.03.2020).

Kurakata, Kensaku : "Une poétique ‘ sans paroles `. Musicalité et impersonnalité chez Verlaine ». In : Europe 85 (avril 2007), 66-73.

Lacoue-Labarthe, Philippe : "Baudelaire contra Wagner ». In : Études françaises 17,3-4 (1981), 2352, https://doi.org/10.7202/036740ar (consultation 30.03.2020).

Landi, Michela : "Un incontro mancato ? Baudelaire e Wagner ». In : LEA - Lingue e letterature d'Oriente e d'Occidente 8 (2019), 1-16, http://dx.doi.org/10.13128/LEA-1824-484x-25590 (consultation 30.03.2020).

Lichtenthal, Julia : « ‘ Un instant à la fois très vague et très aigu... ’. Der Augenblick und seine musikalische Transgression in der Lyrik Paul Verlaines ». In : Herold, Milan / Bernsen, Michael (éds) : Der lyrische Augenblick. Eine Denkfigur der Romania. Berlin : De Gruyter, 2015, 243-261.

Scher, Steven Paul (éd.) : Literatur und Musik. Ein Handbuch zur Theorie und Praxis eines komparatistischen Grenzgebietes. Berlin : Erich Schmidt, 1984.

Touya de Marenne, Éric : «Entre l'éternité et la (post)modernité : les trois musiques de Baudelaire ». In : Dalhousie French Studies 55 (2001), 40-55.

Verlaine, Paul : Euvres poétiques complètes. Éd. Y.-G. Le Dantec et Jacques Borel. Paris : Gallimard, 1962. 